Disciplinar y clasificar: Prácticas de lectura en la Educación Básica Alternativa en Lima

On disciplining and classifying: Reading practices in the Alternative Basic Education in Lima

\author{
Nicolás Vargas Ugalde \\ Pontificia Universidad Católica del Perú \\ nvargas@pucp.pe
}

Recibido: 16-5-2016

Aprobado: 26-8-2016 


\title{
Resumen
}

En este artículo, exploro las prácticas letradas presentes en una escuela pública del programa de Educación Básica Alternativa. El análisis de la información recogida me permite proponer la presencia de dos prácticas centrales. La primera, la lectura como práctica disciplinaria, tiene a la lectura como medio de control y vigilancia de la disciplina de los estudiantes. La segunda, la lectura como práctica clasificadora, observa la organización de una performance particular cuyo valor reside en conocer y reproducir una forma específica de leer oralmente que -asociada a la procedencia geográfica de los estudiantes- dan sentido a una clasificación del dominio de lo letrado. De forma transversal, se propone que estas prácticas se centran en la forma del texto (no en su contenido) y se encuentran vinculadas a factores socioculturales, que a su vez construyen y dan sentido a ideologías acerca de los estudiantes de la noche, que reproducen desigualdades sociales en el ámbito de la educación básica en Perú.

Palabras clave: Educación Básica Alternativa, literacidad escolar, prácticas letradas, lectura, estudiantes jóvenes y adultos

\begin{abstract}
In this article, I explore the reading practices from a public school of the Alternative Basic Education program. Based on the data gathered, I discuss two main practices. In the first one -reading as a disciplinary practice-the event of reading in front of the class turns into a means of controlling and monitoring student discipline. In the second one -reading as a classifying practice-the organization of a particular performance of reading aloud gets associated with the geographical origin of the students and then to their classification within the domain of literacy. Throughout this study, I propose that these practices focus on the form of the text (not on its content) and are linked to socio-cultural factors, which in turn construct ideologies about these young adults studying at night and reproduce social inequalities in basic education in Peru.
\end{abstract}

Keywords: Basic Alternative Education, school literacy, reading, literacy practices, young and adult students 


\section{Disciplinar y clasificar: Prácticas de lectura en la Educación Básica Alternativa en Lima ${ }^{1}$}

\section{Introducción}

En el Perú, se sostienen diversos debates acerca de la calidad de la educación básica, discusiones que se desarrollan sobre todo en el nivel macro de este conjunto de temas (políticas públicas, modelos pedagógicos, evaluaciones de rendimiento, entre otros). Aunque son intercambios de ideas ciertamente necesarios, abordan este tema desde el deber ser. Hay, por otro lado, un menor énfasis en lo que es y, con ello, aludo a lo que sucede en el nivel micro de la realidad educativa: el aula. Este es el espacio en el que se desarrollan las relaciones interpersonales (entre maestros, estudiantes y demás actores sociales involucrados en el proceso educativo) y que -para el interés de este artículo- se realizan a través de lo letrado.

Así, en este artículo, me centro en la actividad de la lectura -en tanto el área de Comunicación es una de las que convoca mayor atención tanto en la academia como en la opinión pública ${ }^{2}$ - y apunto a mostrar cuáles son las prácticas letradas asociadas a ella en el ámbito escolar. En otras palabras, busco responder cómo y para qué se lee en el aula. Específicamente, me ocupo de esta actividad escolar entendiéndola como constituida por un conjunto de eventos letrados -es decir, episodios observables en los que la letra juega un rol importante-, y de prácticas letradas o maneras culturalmente situadas de usar la lectura y la escritura, que siempre están influidas por ideologías sobre diferentes aspectos de la realidad (Barton \& Hamilton, 2004; Bloome, Power Carter, Morton Christian, Otto \& Shuart-Faris, 2005). A partir de esta consideración, brindo argumentos para mostrar dos prácticas letradas recurrentes que constituyen el cómo se emplea la lectura: i) la lectura como práctica disciplinaria y ii) la lectura como práctica clasificadora. Con ellas, finalmente, reviso un conjunto de ideas acerca de un para qué sirve la lectura.

Ahora bien, resulta relevante ubicar esta investigación en una escuela pública, en tanto ella representa un símbolo del Estado que no solo provee de contenidos, sino que se encarga de introducir al estudiante en la sociedad de la que es parte y que, en mi hipótesis de trabajo, contribuye a la construcción de un tipo de ciudadano particular a través de las prácticas letradas que se promueven en el aula. Por supuesto, la formación de la ciudadanía es un proceso complejo y este artículo no busca una explicación concluyente, sino dar luces

1. Esta investigación se basa en mi tesis de licenciatura, cuya versión inicial fue apoyada por el Padet del Vicerrectorado de Investigación y luego sustentada en la PUCP. Agradezco, de igual manera, el reconocimiento otorgado por el concurso nacional de tesis de la Siep.

2. Este interés se ha reflejado en los resultados de encuestas difundidos por los medios masivos de comunicación y, sobre todo, en jornadas de consulta realizadas a nivel nacional con diversos actores de la sociedad (Tovar, 2002). 
sobre algunos aspectos de ella y así aportar a otros estudios al respecto (por ejemplo, ver Ames, 1999). Me interesa el programa de la Educación Básica Alternativa (antes conocido como «el turno de la noche») para el estudio, pues en él se concentra un número marcado de migrantes, quienes son protagonistas de cambios que han reconfigurado la sociedad en años recientes (Degregori, 1987; Matos, 2004 [1984]) y cuya experiencia escolar no ha sido suficientemente atendida.

\section{Enfoque y metodología}

Como es de notar a partir de este planteamiento, mi estudio se ubica en el campo de los estudios socioculturales de la literacidad, que conciben a la lectura como una práctica socialmente situada (Barton \& Hamilton, 2004) y, en ese sentido, busca mostrar lo que va más allá del texto en tanto fenómeno cultural (Street \& Street, 2004). Mi enfoque se vincula con el análisis del discurso y el estudio de los eventos letrados dentro del aula de clase desde una perspectiva microetnográfica (Bloome et al., 2005), que toma en consideración las identidades sociales, las relaciones de poder y los intrincados procesos culturales de la sociedad en un sentido más amplio.

El trabajo de campo se realizó durante cuatro semanas en una institución de Educación Básica Alternativa (Eba) ubicada en Los Olivos, en las cuales se realizaron observaciones de aula de martes a viernes desde el inicio hasta el final de las clases. Las sesiones correspondieron a dos salones: uno de ciclo intermedio (escolaridad primaria incompleta) con doce estudiantes y otro de ciclo avanzado (equivalente a secundaria) con catorce estudiantes. Se llevaron a cabo entrevistas a las docentes encargadas de las aulas observadas en el área de Comunicación, ambas con experiencia sobre todo en Educación Básica Regular en el sector público y en el privado, pero que enseñan en la Eba como una actividad que constituye una fuente adicional de ingresos. La del ciclo inicial es docente de primaria y tiene 48 años, mientras que la del ciclo avanzado es docente del área de Lengua y Literatura de secundaria, y tiene 36 años de edad. Además, durante los descansos, tuve conversaciones informales con los estudiantes de ambas aulas, cuatro y tres, respectivamente. Los datos de las clases y las conversaciones fueron grabados en audio y $\operatorname{transcritos}^{3} \sin$ los nombres de los participantes.

3. Las transcripciones presentadas siguen las siguientes convenciones:

$\begin{array}{ll}= & \text { Sin pausa } \\ (()) & \text { Contexto } \\ {[} & \text { Habla simultánea } \\ (\text { ) } & \text { Ininteligible } \\ \ldots & \text { Fragmento no transcrito } \\ \text { Subrayado } & \text { Énfasis } \\ : & \text { Prolongación del sonido anterior } \\ ? & \text { Entonación ascendente }\end{array}$


Por supuesto, esta constituye una mirada particular con respecto a una realidad bastante compleja. En ese sentido, comparto con Haas y Genishi (2005) el supuesto por el cual el investigador nunca «simplemente» observa un salón de clases, sino que toma una posición y enfatiza en determinados elementos, mientras que deja a otros como contexto. Así, lo que presento es un caso construido a partir del interés de esta investigación en pos de entender y explicar las interrogantes planteadas.

\section{Los estudiantes de la nocturna}

Formalmente, el Ministerio de Educación del Perú (2004) define a los estudiantes de la Educación Básica Alternativa de la siguiente manera:

(a) No se insertaron oportunamente en el sistema educativo.

(b) No pudieron culminar su educación básica.

(c) Requieren compatibilizar el estudio y el trabajo.

(d) Han egresado del Programa de Alfabetización y desean continuar sus estudios.

Es de notar que los dos primeros ítems son enunciados que inician con una negación: «No se insertaron...» y «No pudieron...». Así, la representación que se hace de estos estudiantes consiste en responsabilizarlos por la situación en la que se encuentran por su incapacidad de insertarse y culminar con el proceso educativo. Se desprende de los enunciados que se trata de estudiantes que se encuentran en desventaja y para quienes es necesario un programa de tipo «remedial», que les brinde aquello de lo que carecen; de este modo, la literacidad (leer y escribir) se construye como un producto deseable y es símbolo de poder (Bloome et al., 2005).

A mi entender, esta es una representación del déficit que presupone una concepción de la literacidad como un paquete finito de conocimientos que el Estado entrega a los estudiantes para empoderarlos. Esta concepción se verá relacionada más adelante con ideas concretas en las prácticas letradas, por ejemplo, cuando se solicita a los estudiantes cumplir con una lista definida de formas de leer o cuando solo se privilegia la comprensión literal de los textos, en lugar de promover una relación crítica con los contenidos. Por lo pronto, como plantean Street y Street (2004), los estudiantes son fijados a una identidad particular a través del lenguaje y esto es especialmente relevante, pues se trata de un documento oficial que, como se ha visto, los representa de manera desventajosa.

En el tercer ítem, en cambio, se manifiesta una necesidad que, efectivamente, encontré entre los estudiantes: "compatibilizar el estudio y el trabajo", trabajar en el día y estudiar en la noche. En su mayoría, los alumnos son trabajadores durante el día: ayudantes de cocina en pequeños restaurantes, personal de limpieza en hostales, vendedores ambulantes y, en el caso de las mujeres, 
sobre todo, trabajadoras del hogar. Las mujeres representan el grupo mayoritario en el ciclo inicial, quienes son todas mayores de edad, de aproximadamente veinticuatro años, mientras que en el salón de ciclo avanzado los estudiantes bordean la mayoría de edad, dieciocho años. Trabajo y educación, entonces, son claves en la identidad de estas personas y así lo muestra, por ejemplo, el lema «Estudiar, trabajar y vencer», pintado en una de las banderolas que se prepararon para el desfile de aniversario del colegio. La educación es vista, en este sentido, como un medio para abrirse oportunidades en la sociedad ${ }^{4}$, cuestión que produce sentido al enmarcarlo en lo que otros investigadores han denominado «el mito de la educación» en relación con la ideología del progreso (Ansión, 1995; Montoya, 1990) y que, para nuestro interés, se basa en el supuesto poder de lo letrado (Zavala, 2002).

\section{Las prácticas letradas de la noche}

\subsection{La lectura como práctica disciplinaria}

El primer hallazgo muestra que, en ambas aulas observadas, la lectura de un texto sirve (es decir, se hace algo con él) para establecer (3.1.1.) y vigilar (3.1.2.) un cierto orden durante la clase. A partir de ello, sostengo que esta práctica construye una forma particular de relacionarse con lo letrado y pongo en evidencia que dicha relación se encuentra cruzada por las variables de poder e identidad. Así, esta práctica promueve la construcción de un ciudadano disciplinado.

\subsubsection{El (re)establecimiento del orden}

En esta subsección, muestro cómo la lectura permite a las docentes retomar el control del aula. Por ejemplo, en los tiempos intermedios (cambio de curso, de profesor o luego de los recreos), el aula de clase se presenta como un espacio «libre», en el que los alumnos caminan de un lado a otro, entran y salen, conversan, o estudian por su cuenta; en suma, deciden qué hacer durante ese tiempo y se agencian de lo necesario para hacerlo. Aunque todas estas actividades se encuentran dentro de lo que podríamos esperar en una institución educativa ${ }^{5}$, existe una diferencia cuando las docentes están presentes.

4. Tanto en la consulta nacional mencionada (Tovar, 2002) como en estudios circunscritos a la zona rural (Montero y otros, 2001) se halla testimonio de las expectativas altas presentes en estudiantes y padres de familia.

5. No todas están relacionadas directamente con lo «escolar», también se encuentran actividades comerciales (venta de comida, cosméticos, etc.) realizadas por los propios alumnos. Estas son extensiones de su quehacer diario como jóvenes trabajadores y que -debidamente exploradas- también pueden brindar información sobre el rol de lo letrado. 
En la siguiente interacción, se recoge el momento en el que la profesora ingresa al salón luego de uno de los intermedios mencionados. La profesora se ubica en el escritorio principal y, mientras revisa su material, brinda algunas indicaciones a los estudiantes. Ella pide que le entreguen los cuadernos para revisar las tareas pendientes, pero no todos la escuchan. En este contexto, ella plantea la actividad de lectura.

\section{Extracto 1}

$\begin{array}{lll}1 & \text { Profesora: } & \text { a ver silencio silencio. } \\ 2 & & \text { puedes sentarte? y estar en silencio por favor! } \\ 3 & & \text { garcía estoy hablando. } \\ 4 & \text { ya lee hijo empieza. } \\ 5 & \text { yacumama vamos a leer ya:cu:ma:ma: } \\ 6 & \text { a ver quién está interrumpiendo? } \\ 7 & \text { a ver para sacarlo. }\end{array}$

Lo que encontramos en este fragmento es una voz que reclama para sí el centro de atención: «estoy hablando!». Esta voz les pide a los alumnos «silencio silencio» con la justificación de que es ella -la profesora- quien está hablando. Aunque puede resultar un detalle menor, no deja de llamar la atención la relación que surge entre la voz de la profesora y el silencio de los alumnos. El silencio ha sido reportado en otros estudios como un rasgo de lo que implica un aula ordenada (Ames, 1999). Esto se evidencia en el hecho de que no se les pide que bajen la voz y continúen con sus conversaciones, sino que se les demanda sentarse sin moverse y se corta toda forma de interacción oral. Así, se busca retomar el control (representado por esta voz) y, con ello, se visibilizan las relaciones de poder que se construyen entre los participantes. Nótese que «igarcía estoy hablando!» es un enunciado que -más allá de la distancia y formalidad que plantea el uso del apellido- nos revela el ejercicio de un poder central en las interacciones en el aula: la profesora habla y el alumno escucha. En este sentido, el poder no es una noción estática que se concibe como un producto o una relación a priori, sino que se trata de un proceso que se negocia activamente y varía según el contexto (Bloome et al., 2005).

Lo que me interesa mostrar es que el restablecimiento del orden se encuentra atado a la actividad de lectura. Es decir, la lectura no es una actividad cualquiera que sigue luego de la afirmación de la voz de autoridad, sino que es ella misma (la lectura) la que acompaña y da forma a la autoridad de la profesora. El uso de la lectura no es casual; por ello, debe tenerse en cuenta que la lectura escolar constituye una forma cultural construida históricamente, y que sus prácticas no son las únicas ni las más naturales (Street \& Street, 2004). Salir a leer al frente de la clase no es lo único que se puede hacer con el texto.

Ahora bien, recordemos que el significado se halla definido en las prácticas que lo constituyen; en ese sentido, un ejemplo es el enunciado siguiente 
en la línea 4: «ya lee hijo empieza». La autoridad se manifiesta en la potestad que tiene la docente para asignar el turno de lectura de un texto preestablecido («yacumama vamos a leer ya:cu:ma:ma:»), no de un juego o alguna otra actividad. Así, la docente busca pasar ${ }^{6}$ de un momento «libre» a uno «controlado»y, para ello, usa un mecanismo que incluye a la lectura como pieza central, asociándola a la voz de autoridad. Esta autoridad, además, actúa estratégicamente, pues, como se ve, pasa del uso del apellido del interlocutor "garcía» a «hijo», que es un vocativo que funciona como mitigador de la orden establecida.

Una vez que se tiene un determinado orden establecido -es decir, cuando ya se sabe que el texto será leído y hay un participante con el turno asignado-, la profesora debe asegurarse de que este orden perdure. Así, en la línea 6, la voz de autoridad enuncia en forma de pregunta "¿a ver quién está interrumpiendo?». Por supuesto, esta no espera ninguna respuesta, sino que cumple la función de una advertencia. La maestra levanta la mirada y pasa revista a la clase dirigiéndose simbólicamente a cada uno de ellos. El mensaje es claro: no interrumpan. Así, ambos actores aceptan el hecho de que salir a leer funciona como regulador de la conducta, pues, como se verá, ninguno de ellos lo cuestiona. Se trata, entonces, de un evento en el que se plantea a la lectura como una práctica con la que se busca restablecer la disciplina escolar, instalar reglas y presentarla como una advertencia.

Al final de este extracto, la docente añade en la línea 7: «a ver para sacarlo». De este modo, no solo plantea una advertencia, sino que manifiesta cuál será la consecuencia de romper esta regla: hacer que el infractor salga a leer frente a los compañeros. Esto da pase a un segundo evento de la práctica disciplinaria, puesto que, para poder castigar a los infractores, la docente requiere vigilarlos.

\subsubsection{La vigilancia de la disciplina}

En esta sección, muestro evidencia de una práctica sostenida que no solo recupera el control del aula para la voz de autoridad, sino que vigila la disciplina a lo largo del evento. Con esto, además, demuestro que el uso de la lectura como estrategia de disciplina no es un recurso circunstancial ${ }^{7}$, sino que -a través de la vigilancia- se inscribe en el corazón mismo de la práctica de enseñanza (Foucault, 2009).

6 Puede interesar interpretar el uso de la partícula «ya» desde el uso específico del castellano andino (presente en las docentes y estudiantes). Se sugiere que esta partícula adquiere un valor sustitutorio de un comportamiento anterior por uno nuevo (Cerrón, 2003). Es decir, en este caso, se plantea pasar del caos al orden.

7 Aún si lo fuese, merecería atención en el marco de los recursos pedagógicos con los que cuentan los maestros como parte de su formación. Al respecto se puede consultar Oliart (2011). 


\section{Extracto 2}

\begin{tabular}{|c|c|}
\hline Profesora: & $\begin{array}{l}\text { ahora siguiente este } \\
\text { james. }\end{array}$ \\
\hline James: & nooo! \\
\hline Clase: & $(($ risas $))$ \\
\hline Profesora: & james. \\
\hline James: & miss! \\
\hline Profesora: & $\begin{array}{l}\text { cualquiera voy a llamar }(1.0) \\
\text { que está desatento. }\end{array}$ \\
\hline James: & yo he estado escuchando. \\
\hline Profesora: & $\begin{array}{l}\text { estás jugando. } \\
\text { párate acá. }\end{array}$ \\
\hline & en el centro. \\
\hline Clase: & $(($ risas $))$ \\
\hline
\end{tabular}

En este fragmento, interesa mostrar cómo la profesora designa el siguiente turno cuando termina una intervención. Recordemos que la actividad de lectura ha sido planteada como un castigo para los que interrumpen $y$, en consecuencia, es de notar que sea la docente quien determina a quién le toca leer. Para esta decisión, solo emplea su criterio y no explica las razones. En algunas pocas ocasiones, apeló también al método de la lista, que no difiere en mayor medida en cuanto a la arbitrariedad de la decisión. No se encontró ocasión en que se preguntase a quién le gustaría salir o se empleara algún método alternativo (y creativo) para hacer menos aleatoria la sucesión de turnos, o -en el mejor de los casos- relacionar la sucesión de turnos con algún criterio pedagógico.

En este punto, encuentro coincidencias con lo que señala De los Heros (2012) acerca de la ideología del secreto. La autora plantea la existencia de prácticas regulares por las que el poder se ejerce sobre el otro sin dar explicaciones sobre ninguna de sus decisiones, desde las más simples hasta las más complejas. Ninguno de los sujetos cuestiona estas formas y -desde una perspectiva más amplia- esto tiene implicancias en el ejercicio de la ciudadanía. Se ha visto en otros estudios que la socialización temprana con la autoridad escolar se vincula con relaciones posteriores de otro tipo, como la política (Vásquez \& Martínez, 1990), lo cual es especialmente particular en el caso de estos alumnos que, en su mayoría, son adultos y están siendo formados para mejorar su vida cotidiana, tal como se establece en la definición oficial de la Eba.

Ahora bien, en la línea 7, tenemos una muestra del ejercicio de este poder en la respuesta por parte de la profesora: «cualquiera voy a llamar». Esta intervención va de acuerdo con la idea de tomar una medida sin dar explicaciones, que he relacionado con la ideología del secreto. Así, el «cualquiera voy a llamar» deja abierta la posibilidad incierta de ser citado y solo es en la siguiente intervención, en la línea 8 , mediada por una pausa de un segundo, que se 
puntualiza: «que está desatento». En ese sentido, la única explicación que cabe en esta situación es la que concibe a la lectura como una práctica de control: sale el desatento. Ya no solo se restablece el orden, sino que este se mantiene con la amenaza de la lectura.

Para ahondar en este argumento, propongo introducir la idea de que el control de la disciplina ejercido a través de esta actividad tiene en este momento un componente fundamental: la vigilancia ${ }^{8}$. Esta es una idea poderosa en tanto no estamos frente a un par de hechos aislados en los que el docente plantea la idea de castigar el mal comportamiento, sino que, al encontrar estas manifestaciones de indisciplina presentes a lo largo del evento letrado, se visibiliza cada vez más un sistema de control que se sirve de la lectura como herramienta.

Hacia el final del extracto, se confirma cómo es que opera la mirada de la profesora. Ante la réplica del alumno (línea 9) «yo he estado escuchando», la voz de la autoridad responde "estás jugando». En este punto, se observa un correlato gramatical relevante para la explicación, pues, en los tiempos verbales empleados, la idea de la vigilancia se refuerza como estrategia constante: la conjugación del tipo «estar + gerundio» señala que la acción (en este caso, la infracción cometida por el alumno) se está realizando en ese momento. La vigilancia de la docente es efectiva, incluso cuando no está mirando directamente a los estudiantes. En este sentido, propongo que no solo el castigo, sino que el hecho mismo de ser visto constantemente es lo que mantiene al individuo en el sometimiento (Foucault, 2009).

Presento el siguiente extracto para consolidar el argumento que sostengo sobre la práctica disciplinaria y dejar en evidencia cómo es que opera. Una alumna que lee en voz baja logra completar las oraciones con bastante dificultad y, durante su intervención, se evidencian largos silencios. En ese contexto, se produce esta interacción:

\section{Extracto 3}

1 Alumno: no sabe leer.

2 Clase: ((risas))

3 Profesora: ya ahorita sale.

$4 \quad$ ya vas a leer tú ahorita.

Un alumno de entre el grupo afirma «no sabe leer» usando un tono de broma y que, a la vez, -de manera muy dura- hace explícito lo evidente sobre la performance de su compañera. Al comentario de la línea 1, la clase responde con una risa general. Precisamente, esa risa es la que permite comprender lo

8. Foucault (2009) realiza un repaso por la construcción cultural del orden y señala que la escuela es una institución celadora de él. En la dinámica de esta, estas formas se ponen de manifiesto, por ejemplo, a través de la vigilancia. 
enunciado en la línea 1 , en la que la identidad de la compañera es construida como la de un sujeto carente, objeto de burla. Por supuesto, más allá de la alumna en cuestión, lo que se estigmatiza es la forma de leer con la que se le identifica.

Ahora bien, ninguno de los alumnos manifestó desacuerdo y tampoco la profesora se ocupó en atender esta evidencia (la alumna tenía serias dificultades para leer o, en todo caso, para hacerlo frente a la clase). La profesora respondió primero de manera general «ya ahorita sale» (línea 3) sin voltear a mirar, anticipando que sería cualquiera quien saldría. Solo después volteó para ubicar al alumno. La profesora conoce a sus alumnos, incluso por la voz, así que no le fue complicado ubicar al autor y continuar "ya vas a leer tú ahorita» refiriéndose directamente a uno de ellos. Con esto último, resulta claro que la vigilancia es una estrategia que tiene a un agente (la voz de autoridad representada por la profesora) bastante bien entrenado para su ejecución, pues los reconoce incluso sin necesidad de observarlos. Así, los estudiantes se encuentran siempre sometidos.

Hasta el momento, se observa, entonces, un evento inicial en el que se presenta a la lectura como un medio para restablecer el orden de la clase (extracto 1) y se la plantea como una amenaza velada. Luego, en los extractos siguientes (2 y 3), se muestran momentos definidos en los que se hacen explícitos el mecanismo de control y, sobre todo, la idea de una estrategia de vigilancia constante. Queda claro, en esta sección, que la lectura no es una «inocente» actividad escolar, sino que -más bien- funciona como un mecanismo de coerción $\mathrm{y}$, con ello, se ve cómo juega un rol central en la formación de un estudiante disciplinado.

\subsection{La lectura como práctica clasificadora}

Se sabe que siempre se lee de una manera particular en cada situación (Zavala, Niño-Murcia \& Ames, 2004); sin embargo, no siempre damos cuenta de cuáles de esas formas particulares son las hegemónicas en un ámbito como el educativo. En esta sección, propongo delinear una forma específica de hacer con la lectura (que llamaré performance letrada), cuyo cumplimiento le sirve a la maestra para clasificar a los estudiantes en dos grupos: quienes sí saben leer y quienes no saben leer. Luego, argumento que esta práctica es asociada a rasgos de los estudiantes, que tienen que ver con lo social (Gee, 1999) y con lo geográfico (Orlove, 1993).

\subsubsection{Las condiciones de la clasificación}

En los casos observados, saber leer es saber performar la lectura y, para, ello he identificado un conjunto de características que las docentes exigen que los estudiantes cumplan. Las más resaltantes son leer en voz alta, la pronunciación del léxico, la entonación, la postura del cuerpo, entre otras de menor 
frecuencia. Estas condiciones que se erigen como normas han sido socialmente construidas como las correctas y su presencia en el aula asegura su pervivencia a través de la repetición.

En la siguiente interacción, se muestra a la profesora brindando indicaciones a una alumna (Liz) que se encuentra ya frente al salón de clase.

\section{Extracto 4}

1 Profesora: si tienes voz todavía bajo y te pones allá.

2 Liz: en el centro del agua (...) burbujas pareció.

3 Profesora: parecía.

4 Liz:

5 Profesora:

parecía responderle responderle y la empeci [empeci

6 Liz: [empecinada

7 Profesora: =qué?

8 Liz: ((se queda en silencio))

9 Profesora: a ver.

10 el pecaví pecaví es un pez una animalito.

11 Liz:

12 Profesora:

el pecaví. genaro valdivia ((se queda en silencio))

13 Liz:

14 Profesora:

15 Liz:

16 Profesora: amonestó severamente. amonestó severamente. voy seguir? sigue. cuidado con salir hasta [que ya rosa a ver tú.

[hasta ahí nomás cuidado con salir ahí quiere decir que su papá le dejó dice unas tostadas unas este hormigas para que pueda comer su hijo. y luego le dijo que por favor no saliera de la choza no? hasta que él regrese.

Antes de la intervención de la profesora, la alumna se encontraba ubicada junto a la pizarra, hacia el lado más próximo a su carpeta, incómoda con la tarea asignada. Desde ahí, leía el fragmento del texto encargado. En la línea 1 , se le hace explícito a la alumna que tener voz baja es una desventaja y que, sabiendo eso, ella debería buscar remediar la situación. Aquí «todavía» (línea 1) funciona enfáticamente y refuerza el supuesto de que la lectura debe hacerse en voz alta, con una determinada entonación.

En otros momentos similares a este, también es posible hallar voces correspondientes a alumnos que señalan «no se escucha» o «iya salió?» siempre en tono de broma, con lo cual refuerzan la idea de que hay una forma de leer y que la compañera no la está perfomando eficientemente. Esto, también, nos muestra que se trata de un uso extendido, pues tiene resonancia incluso en las bromas de los estudiantes. Se produce en ello una complicidad entre la maestra 136 I y los otros alumnos frente a quien está en el turno de lectura. Precisamente, 
esto es lo que constituye una práctica, la idea compartida sobre una forma reconocida por los miembros de la comunidad.

Por su parte, el léxico también constituye un punto central que, según se ve en las líneas 4, 6 y 11, no es aprovechado para informar a los alumnos sobre el significado de una nueva palabra, de modo que se enriquezca su vocabulario, sino que solamente se corrige o aclara la pronunciación. Esto se debe a que la forma de pronunciar se identifica y se enseña más fácilmente. Se trata, entonces, de una instrucción bastante específica sobre leer exactamente como se encuentra en el texto. Ahora bien, no se trata simplemente de afirmar que la profesora sigue a pie juntillas el texto sin razón alguna, sino que propongo que hay detrás una estrategia que explico con la noción de prácticas seguras.

Las prácticas seguras son aquellas por las que estudiantes y maestros buscan preservar su dignidad escondiendo el hecho de que poco o ningún aprendizaje se lleva a cabo (Hornberger \& Chick, 2001). En palabras de las autoras citadas, un ejemplo claro de ello es el recurso de responder en coro, que se halla en las aulas peruanas y, por supuesto, en las clases que observé:

We conclude that the chorusing, here as in the Peruvian case, functions principally to give students opportunities to participate in ways that reduce the possibility of the loss of face associated with providing incorrect responses to teacher elicitations or not being able to provide responses (Hornberger \& Chick, 2001, p. 35).

Así, en el caso de las líneas 2 y 4, por ejemplo, encontramos oportunidades en las que la lectura no es realizada fluidamente debido a palabras que resultan poco o nada familiares para la alumna. La profesora la socorre pronunciando las palabras completas (líneas 3 y 5), las enuncia para hacerlas familiares, pero no dice nada sobre los significados o los contextos en los que se están empleando. Encontré esto de manera sistemática y resalto que se trata de palabras cuyos significados no tienen referentes inmediatos o concretos, y que, más bien, exigirían un ejercicio de abstracción para ser explicados. Precisamente, por ello, sugiero que una razón para pegarse al guion y exigirlo como una instrucción de la performance letrada es evitar entrar en un terreno en el que se expongan tanto la alumna como la profesora. En concreto, la profesora se vería retada a usar recursos pedagógicos que le permitan explicar los conceptos nuevos encontrados en las lecturas; la alumna, por su parte, tendría que ir más allá de la repetición de una cadena sonora. Esta instrucción es, entonces, una práctica segura en la que ninguna de las participantes arriesga su imagen; ninguna de las dos se exige más allá del conocimiento de una pieza léxica.

Tomemos un ejemplo que sirve de contraste, en el que la docente no solo aclara el léxico, sino que lo explica. De la línea 6 a la 10, sobre el "pecaví», la docente va más allá de la repetición de la palabra y señala: «un pez una animalito». No obstante, esta explicación realmente no requiere mayor esfuerzo de elaboración conceptual; la docente recurre a un referente concreto que 
simplifica el conocimiento. En este caso, ambas, profesora y alumna, no tienen problema en reconocer un animal y avanzan así sobre terreno seguro. Este tipo de prácticas deben llamar la atención en tanto se encuentran naturalizadas y extendidas en el sistema educativo, tal como se ha encontrado en otras investigaciones (De los Heros, 2012; Hornberger \& Chick, 2001).

Para continuar con el tratamiento del léxico, introduzco un extracto que busca reafirmar la idea de que existe un interés marcado en la forma fónica, es decir, en la pronunciación como condición de la buena performance de la lectura.

\section{Extracto 5}

1 Alexis: en una choza amazónica a orillas del río ucayali genaro valdivian vio 2 con sorpresa que sus previsiones se acababan (...) e ex [ex

3 Profesora: [exquisito

4 Alexis: ah exquesito.

5 Profesora: ex:qui:si:to:

6 Alexis: exquesito.

7 Alexis: ((más adelante en la mismaintervención) $)$ sonrisalleno de orquideyas=

8 Profesora: =orquídeas

9 Alexis: orquídeas

Es preciso notar que, luego de la intervención de la profesora en la línea 5 , la alumna retoma a partir de la línea 6 y continúa leyendo el cuento. La maestra no vuelve a intervenir, sino hasta la siguiente palabra (en la línea 8), de cuya forma fónica también se ocupa. De igual manera, la alumna escucha la corrección sobre la pronunciación del léxico, retoma la lectura en la línea 9 y continúa leyendo el cuento. Esto continúa así hasta el fin de su turno o, en general, hasta que aparezca una nueva pieza léxica cuya pronunciación requiera la intervención de la docente.

Ahora bien, en ambos casos (líneas 4 y 7), se ve con claridad que la alternancia vocálica del castellano andino podría jugar un rol explicativo en cuanto a la pronunciación que los alumnos realizan; sin embargo, no he registrado ninguna mención a ello directamente. Con la intención de aclarar el tema, indagué con los profesores sobre el tema de variedades regionales incorporadas en la currícula de clase, y recogí algunas opiniones contradictorias sobre el valor de lo cultural y -a la vez- el "problema» (extracto 6) que ello significa en Lima.

Sobre esto último, incluyo el fragmento de una conversación que sostuve con una docente. En ella, tratábamos el tema de la clasificación de estudiantes y apareció el quechua como factor explicativo. 


\section{Extracto 6}

1 Nicolás: y cómo ve esa relación entre lo que leen y escriben? es diferente?

2 Profesora: sí por ejemplo ellos como están acostumbrados de repente le han 3 enseñado quechua pues.

4 Nicolás: ah claro.

5 Profesora: usted ha visto quechua?

6 Nicolás: sí sí.

7 Profesora: el quechua es diferente a lo que nosotros enseñamos en castellano. 8 como ella sabía quechua antes me contó y por eso no escribía bien y ahora ha mejorado pero en lectura y eso se enseña en primaria. está atrasada.

11 Nicolás: entonces le parece que el quechua no los ayuda?

12 Profesora: no los ayuda pue porque les hace equivocar en la manera de escribir no? ((dirige la mirada a una alumna)) pero según dice nosotros también debemos saber quechua porque hay alumnos que de repente vienen acá a estudiar y hablan quechua y uno les puede enseñar en quechua.

De esta interacción, quisiera resaltar dos puntos. El primero es que, efectivamente, el quechua es visto como una interferencia que, por ejemplo, explica la diferencia que existe entre la lectura y la escritura de un mismo estudiante (líneas 2 y 3). Una alumna, incluso, puede lograr cumplir con las condiciones de la performance letrada y, en ese sentido, mejorar su lectura (línea 9), pero, como se lee en la línea 8 , la escritura seguirá siendo una tarea pendiente por el hecho de saber quechua «antes». No obstante, no pretendo presentar la existencia de un conjunto de ideas definido y cerrado sobre el rol del quechua en la educación de estos estudiantes, sino que, más bien, como segundo punto, quisiera relativizar la postura de la profesora. Como se ve de la línea 12 a la 16, en un mismo enunciado, podremos encontrar dos ideas que se contradicen: una que representa a esta lengua de manera negativa en tanto el quechua «les hace equivocar», y una segunda idea que la revalora y la representa de manera más bien positiva, pues «nosotros también debemos saber quechua». Estas valoraciones sobre el quechua no son nuevas ni particulares de estas aulas; se puede ver en otros estudios esta actitud con respecto a esta lengua (Cueto, Andrade \& León, 2003) y las concepciones ambivalentes en torno a ella (Zavala, Mujica, Córdova \& Ardito, 2014).

Esto último es especialmente interesante, pues me permite dialogar con autoras como De los Heros (2012), que encuentra ideologías contrapuestas en las aulas con respecto al estándar, y Zavala (2012), que propone la presencia de ciertos dilemas ideológicos entre los maestros de Educación Intercultural Bilingüe. Con ellas, coincido en que, más allá de las particularidades de los casos que cada investigadora analiza, estos hallazgos posibilitan problematizar las 
nociones sobre las ideologías, en la medida que no se trata de sistemas completos, sino que son más bien fragmentados y, como en este caso, incluso, contradictorios. Hay en ello una lucha implícita de diferentes sentidos en pos de hegemonía, lo cual abre la puerta a la posibilidad de instalar nuevas ideas en las que, para seguir con el caso del quechua, la lengua originaria deje de ser vista como un obstáculo para el aprendizaje de los estudiantes.

Más allá de estas consideraciones, busqué contrastar las declaraciones de las docentes con el material que oficialmente se destina para la Educación Básica Alternativa, pero no existe ningún tipo de guía o libro de texto que las docentes usen o tenga a su disposición para llevar a cabo las clases. Según lo que ellas me reportaron, depende de su iniciativa, de la experiencia que tengan, del material conseguido por cuenta propia, etc. De este modo, encuentro profundamente preocupante ya no solo la contradicción a la que hacía referencia en cuanto a la valoración de la lengua originaria, sino la completa invisibilización de estas características presentes en los estudiantes de la Eba. Una forma de reproducir las condiciones de injusticia de un sector de la población mediante la educación es, precisamente, haciendo invisible su propia historia (Anyon, 1981).

En los extractos destacados en esta sección, se ha procurado mostrar la atención que toman las docentes sobre la forma de performar la lectura y, en particular, se ha visto que las dificultades identificadas son asociadas al quechua.

\subsubsection{La clasificación según el lugar de origen}

A continuación, se observará que el cumplimiento de la performance letrada visto en el acápite anterior es usado para generar una clasificación de estudiantes: los que sí saben leer y los que no saben leer. Propongo que lo que se encuentra detrás de esta división no es exclusivamente una consideración vinculada a la lectura misma, sino que las docentes realizan una asociación con otros rasgos de los estudiantes.

El siguiente extracto es parte también de las conversaciones que mantuve con las maestras en los intervalos entre clases, precisamente minutos después de ocurridos los eventos letrados en discusión. Esta inmediatez fue productiva en tanto la experiencia estaba fresca en la memoria de las docentes, y ello permitió un acceso menos trabado a las ideas y valoraciones acerca de esas prácticas. Así, en el extracto 7, una de las maestras comenta sobre las diferencias que ella encuentra en su aula y alude al lugar de origen de los estudiantes como un factor a tomar en cuenta. 


\section{Extracto 7}

1 Nicolás: la minoría es de lima la mayoría es?

2 Profesora: la mayoría son del campo.

3 Nicolás: y eso le parece que influye en algo?

4 Profesora: sí influye para hablar se sienten mal no?

6 qué dirán si de repente hablo mal. en eso.

Nicolás: $\quad$ cómo cree que se dan cuenta los otros alumnos?

Profesora: los conocen pue como ellos conversan entre ellos.

Nicolás: y usted cómo se podría dar cuenta de que alguien está hablando mal?

0 Profesora: por el dejo.

11 Nicolás: ah ya.

12 Profesora: yo los saco porque tengo años acá.

Nicolás: ajá: a los limeños se les conoce porque son más vivos.

Profesora: a los de la sierra son más tímidos. a los de la selva porque son más fuertes como ella. es diferente su forma de ser total diferencia.

\section{Nicolás: ajá::}

Profesora: por eso yo les hablo yo les llamo la atención a los que son de lima pues. ustedes tienen su mamá debieran ser mejores que ellos en cambio ellos ponen más de su parte en cambio ustedes? bueno les llamo la atención cuando no les veo atentos miren ellos vienen de la sierra estudian y trabajan al mismo tiempo y ellos mismos hacen su gasto. en cambio tú tienes más apoyo y trata de mejorar ellos dos han salido jalados.

Nicolás: ah sí?

Profesora: sí flojos son.

Nicolás: $\quad$ y usted identifica quiénes son de la sierra de la selva y los saca.

Profesora: lo trato de sacar más al que no sabe. por ejemplo ella no sabía leer y ya ha aprendido bastante. no sabía leer nada el año pasado le dije que lea.

lee bastante y ha leído seguramente.

Nicolás: entonces sí le parece que mejoran?

Profesora: ha mejorado ella bastante. no sabía leer nada nada ni el diez por ciento de lo que leer pero hasta ahorita en escribir sí se equivoca. ((mirando a la alumna)) la r no no? después o antes pero en lo que es leer sí.

La riqueza de este diálogo nos permite comprender varios aspectos. Por lo pronto, lo que me interesa resaltar es la idea de que la maestra evalúa la lectura 
de sus estudiantes (y a ellos mismos), a partir de variables que están relacionados con factores socioculturales. Esto implica que una determinada manera de leer (la perfomance letrada) indexa un tipo de identidad. Se construye, de este modo, un Discurso (con D mayúscula en el sentido de Gee, 1999) acerca de la lectura que se caracteriza por incorporar otros elementos además del lenguaje en uso. Como ya se ha señalado, la lectura no existe aislada del contexto en el que se emplea.

El lugar de origen es una variable sociocultural que la docente entiende como un problema en tanto generaría en los estudiantes una falta de confianza al momento de afrontar la lectura. El saberse no limeños, en términos de la profesora, enfrenta a los estudiantes de provincia a un "qué dirán» cada vez que les toca leer en público, una suerte de evaluación por parte de la docente y de sus pares. En la práctica, estas ideas expuestas por la docente tienen como resultado una clasificación entre i) unos alumnos a los que les pide leer para que aprendan a leer y ii) otro grupo de estudiantes para los que leer en público es menos imperioso, pues, para el caso de ellos, existen otros factores (por ejemplo, la flojera) que serían los que explican su relación con los textos. Es decir, ambos tienen el mismo «problema» (no cumplen con las instrucciones de la performance letrada), pero la maestra otorga diferentes explicaciones para tal problema. Precisamente, en esa asociación, se produce una diferencia sustantiva entre sus estudiantes: los que sí saben leer y los que no. Así, para el interés de este artículo, se construye discursivamente una distinción que, a través de lo letrado, indexa una división entre dos grupos de personas, los de Lima y los de provincia. Por supuesto, uno de ellos se convierte en una suerte de modelo de estudiante, mientras que el otro, de nuevo, se representa de manera deficitaria.

En un sentido más amplio, la maestra reproduce un discurso bastante extendido en nuestra sociedad, la racialización de la geografía (Orlove, 1993). Me refiero a valoraciones asociadas al lugar de origen de los sujetos, tanto positivas como negativas. En términos históricos, Orlove lo propone de la siguiente manera:

The principal contribution of geographical representation of Indians to the national elite discourses, though, was the association between the highlands and the Indians. Precisely like the highlands, the Indians became an «obstacle» which impeded «integration» and thus retarted national «progress» (1993, p. 327).

Ser de provincia, entonces, constituye un «obstáculo» para el aprendizaje y el progreso educativo de los propios estudiantes que -no se debe perder de vista- son ciudadanos accediendo a un servicio del Estado. Con esto, se devela cómo las estructuras de la sociedad atraviesan las paredes de la institución educativa y dejan al descubierto la falsa noción por la cual la escuela es entendida como una suerte de «burbuja», un supuesto espacio aislado en el que se 
prepara individuos para la sociedad y cuyas actividades son «neutrales». En mi propuesta, en cambio, se entiende a los estudiantes y maestros no solo como actores escolares, sino también como actores políticos inmersos en la sociedad (Mujica, 2005), más aún cuando los participantes de esta investigación bordean la mayoría de edad y, efectivamente, son parte de la ciudadanía política y económicamente activa. En ese sentido, suscribo a Trapnell, quien propone abordar la escuela desde una perspectiva crítica que cuestiona su pretensión de presentarse como natural y universal (2011, p. 158).

Ahora bien, como hemos visto hasta ahora, las valoraciones construyen sujetos delimitados - en este caso, «alumnos provincianos tímidos que no saben leer» $y$ «alumnos limeños que saben leer pero que son flojos»-, pero esto no se queda en el plano de las ideas, sino que, con esas definiciones, la maestra clasifica a los alumnos para asignarles roles y actividades específicas en la dinámica de la clase. Es decir, no se trata solamente de una «representación mental» que los profesores se hacen del grupo humano con el que trabajan, sino que ello les permite, en primera instancia, organizar a los estudiantes a su cargo y, en segundo término, desarrollar estrategias particulares para que sus estudiantes «superen» exitosamente las «taras» identificadas. $\mathrm{Al}$ respecto, veamos la afirmación de la profesora (de las líneas 33 a la 35) en la que señala que su alumna «ha mejorado bastante». Con ello, valida la clasificación y las estrategias que aplicó durante el año. Se observa, pues, cómo las prácticas les dan significado a las ideas de las docentes recogidas en el extracto presentado.

Para complejizar este hallazgo, presento la siguiente interacción, en la que se continúa conversando acerca de los estudiantes «de provincia». En este fragmento, se evidencia cómo se asocia la dificultad para la lectura con la timidez y el temor. Para conectar con la argumentación anterior, mi propósito es explorar la idea de que un factor externo y ajeno a la voluntad del alumno -como el lugar de origen- se halla finalmente asociado a características intrínsecas de la personalidad del alumno (timidez, temor). Así, el "problema» con la lectura pasa de ser causado por factores externos (como el lugar de origen) a ubicarse dentro del alumno. En otras palabras, los agentes del sistema educativo contribuyen a interiorizar el problema en el estudiante y hacerlo responsable por ello. A continuación, mostraré un panorama más completo de las consecuencias de estas asociaciones entre una determinada forma de leer y los factores a los que esta forma es indexada. Estas consecuencias, a la luz de otras investigaciones, coadyuvan a la reproducción de un determinado orden de cosas que no favorece al estudiante. 


\section{Extracto 8}

1 Nicolás: son tímidos en general o por usted cree alguna razón?

2 Profesora: dos tres que en sí no tienen vergüenza pero la mayoría sí tímidos.

3 Nicolás: pero eso se deberá: a qué se deberá eso?

4 Profesora: no pronuncian bien o de repente temor a que no se pueden grabar bien las palabras yo le digo qué importa que se graben todo pero que salgan.

En este fragmento, se observa cómo el ser tímido (línea 2) es presentado como una desventaja para realizar una adecuada performance de la lectura. Esa relación con la personalidad de los alumnos ubica en el interior mismo de ellos las razones por las que no pueden cumplir con la tarea asignada. En esa línea, se puede observar uno de los lemas en las banderolas (preparado también para el desfile de aniversario) que encontré: "No hay sino una regla verdadera de progreso: supérate a ti mismo». Para explicar este vínculo, quisiera traer a colación los hallazgos publicados por Zavala y Córdova acerca de un fenómeno distinto como el motoseo, pero que finalmente trabaja bajo el mismo supuesto: el problema «se supera solamente con la voluntad del sujeto"» (2010, p. 58). Este supuesto, entonces, instalaría la responsabilidad dentro del alumno, haciéndolo culpable de su propio fracaso.

Sobre esto último, una conversación con una estudiante es particularmente esclarecedora. La alumna se acercó mientras me encontraba conversando con otras de sus compañeras, e hizo una serie de preguntas; de ellas, "¿por qué no entiendo nada de lo que leo?, ¿qué me estará faltando o qué tengo que comer?» llamó la atención de todos. Aunque en el momento causó algunas risas de parte de sus compañeras, pronto nos dimos cuenta de que se trataba de una pregunta que llevaba una preocupación honesta sobre el tema. Las demás estudiantes se mostraron de acuerdo en que la lectura era un tema de preocupación para ellas. A través de ello, se evidencia que también en estas conversaciones informales el discurso gana terreno dentro del sentido común de los propios alumnos; es tal su hegemonía que convence a los estudiantes de verse ellos mismos como sujetos carentes.

En términos más generales, propongo una conexión con una investigación ya clásica en la que se ahonda en relación con las características de los estudiantes (Willis, 1977). En ella, el autor busca los mecanismos por los cuales menores de edad de clase trabajadora consiguen trabajos típicamente asociados a la clase trabajadora; de este modo, perpetúan un ciclo naturalizado en el que cada quien realiza la labor «que le corresponde». La investigación encuentra que la escuela cumple un rol fundamental en este proceso, y halla diversas prácticas y discursos como el que he explorado en cuanto a la individualización de la responsabilidad (que se pone de manifiesto en la forma como la alumna plantea su problema). En ese sentido, un lema aparentemente positivo como "supérate a ti mismo», asociado con prácticas letradas como las estudiadas en este artículo, pueden devenir en lo que el autor señala como una noción del estudiante como un sujeto 
escindido: por un lado, los profesores reconocen valores en los alumnos (en cuanto al esfuerzo que hacen al estudiar luego de todo un día de trabajo) y, por otro lado, generan que estos interioricen una culpa que los convierte en sus propios peores enemigos. En ese sentido, no debería sorprender que una de las alumnas tenga preguntas como las señaladas en el párrafo anterior, en las que se muestra que esas representaciones de los alumnos como sujetos deficitarios se hallan ya instaladas en ellos mismos.

Ahora bien, no perdamos de vista que estas valoraciones se realizan sobre la base de lo letrado, de una visión particular (y extendida) de lo que es saber leer. Esto, en mi análisis, solo reproduce un sistema perverso por el cual la promesa de la Eba no brinda oportunidades, sino que mantiene (o incrementa) la brecha.

\section{A modo de conclusión: ¿Para qué sirve la lectura?}

La primera práctica analizada tiene a la lectura como medio para restablecer el orden y vigilar la disciplina de los estudiantes. En cuanto a la segunda, observé la organización de una práctica performativa cuyo valor reside en conocer y reproducir una forma específica de leer, con la cual se clasifica a los estudiantes por su lugar de origen. De forma transversal, he mostrado que estas prácticas se centran en la forma del texto (no en su contenido) y se encuentran vinculadas a factores socioculturales, que, a su vez, construyen y dan sentido a ideologías acerca de los estudiantes.

Se comprueba con ello que, efectivamente, la letra escrita es un instrumento, pero no lo es en el sentido de un objeto cualquiera que se usa en función de propósitos ajenos a su propia naturaleza, sino que se instala en el entramado mismo de la enseñanza y contribuye a darle forma. Con ello, lo que he demostrado es que la lectura se ha establecido ella misma como parte de un determinado orden que, como se ha visto, reproduce las desigualdades haciendo partícipe de ello, incluso, a los propios estudiantes. Las risas registradas en los extractos 2 y 3 , por ejemplo, muestran una recepción en clave de humor del castigo, con lo cual validan y celebran el rol que les toca cumplir. Nadie reclama. En ese sentido, la hegemonía de este discurso se consolida al lograr inducir al grupo subordinado a verse a través de la mirada del grupo dominante (Luykx, 1998).

Así, una lectura conjunta de estos hallazgos me permite afirmar que se trata de un orden de cosas injusto, que se perpetúa a través de prácticas cotidianas que mantienen a los alumnos de la noche en el mundo de la noche -para usar el horario en el que asisten a clases como metáfora-. Las implicancias de esto se relacionan con oportunidades limitadas para acceder a los recursos del Estado y la persistencia de una promesa incumplida en cuanto a lo que se espera de la educación por parte de un sector de la población de escasa movilidad social.

Es relevante recordar que, para el caso de la Educación Básica Alternativa, según su reglamento de funcionamiento, la finalidad es introducir a los 
estudiantes en la sociedad a través de la mejora de los desempeños que demanda la vida cotidiana. Con esto en mente, exploré cómo es que estas prácticas nos brindan información más allá de la interacción en el aula y he revisado sus implicancias en la participación ciudadana de estos estudiantes para demostrar que no concuerdan con aquellas propuestas desde el Ministerio de Educación. Se podría afirmar que, en ese sentido, me he encargado de aquello no explícito y que puede identificarse con la noción de «currículo oculto», que resulta útil cuando se trata de desentrañar aquello que se transmite a través de reglas subyacentes que estructuran las relaciones sociales (Torres, 1998). En ese respecto, he sugerido la pervivencia de ideologías fuertemente arraigadas en la sociedad: las formas de toma de decisión contrarias a la intención participativa del programa educativo, la relación con la autoridad a través de prácticas autoritarias en el aula, la estigmatización social vinculada a atributos racializados como el lugar de origen, entre otras prácticas que pueden continuar explorándose con el propósito de hacerlas explícitas y desterrarlas.

Quiero finalizar resaltando que es fundamental detenernos a pensar en la manera en que se producen estos primeros vínculos con lo letrado, pues, como se sabe, el poder simbólico de la lectoescritura excede los límites de la escuela. La literacidad, en esa medida, es fundamento del moderno Estado-nación y de las instituciones que gobiernan la vida en sociedad. Por ello, lo peligroso de estas formas de relacionarse con lo letrado es que sean entendidas como la única posibilidad. A partir de esta realidad, queda la constante tarea de pensar en las alternativas que pueden ofrecerse desde el campo de la pedagogía.

\section{Nota biográfica}

\section{Nicolás Vargas Ugalde}

Es licenciado en Lingüística y asistente de docencia en la PUCP. Trabaja en la gestión de proyectos de innovación, y la capacitación de docentes en el uso de tecnologías para el aprendizaje en los niveles técnico y universitario. Investiga temas de lenguaje, tecnología y educación. 


\section{Referencias}

Ames, P. (1999). "El poder en el aula: un estudio en escuelas rurales andinas". En Tanaka, Martín (ed.), El poder visto desde abajo: democracia, educación y ciudadanía en espacios locales. Lima: IEP.

Ansión, J. (1995). Del mito de la educación al proyecto educativo. En G. Portocarrero \& M. Valcarcel (eds.), El Perú frente al siglo XXI. Lima: Pontificia Universidad Católica del Perú.

Anyon, J. (1981). Social class and social knowledge. Curriculum Inquiry, 11(1), 3-41.

Barton, D. \& Hamilton, M. (2004). La literacidad entendida como práctica social. En V. Zavala, Niño-Murcia, M. \& Ames, P. (Eds.) (2004), Escritura y sociedad. Nuevas perspectivas teóricas y etnográficas. Lima: Red para el Desarrollo de las Ciencias Sociales en el Perú.

Bloome, D., Power Carter, S., Morton Christian, B., Otto, S. \& Shuart-Faris, N. (2005). Discourse analysis and the study of classroom language and literacy events. Londres: Lawrence Erlbaum Associates.

Cerrón Palomino, R. (2003). Castellano andino. Aspectos sociolingüísticos, pedagógicosygramaticales. Lima:FondoEditorialdelaPontificiaUniversidad Católica del Perú.

Cueto, S., Andrade, F. \& León, J. (2003). Las actitudes de los estudiantes peruanos hacia la lectura, la escritura, la matemática, y las lenguas indígenas (Documento de Trabajo, 44). Lima: Grade.

Degregori, C. I. (1987). Conquistadores de un nuevo mundo: de invasores a ciudadanos en San Martin de Porres. Lima: Instituto de Estudios Peruanos.

De los Heros, Susana (2012). Utopía y realidad: nociones sobre el estándar lingüístico en la esfera intelectual y educativa peruana. Lima: Instituto de Estudios Peruanos.

Dyson, A. H. \& Genishi, C. (2005) On the case. Approaches to language and literacy research. New York: Teachers College Press.

Foucault, M. (2009). Vigilar y castigar: nacimiento de la prisión. México D.F.: Siglo Veintiuno.

Gee, J. P. (1999). An introduction to discourse analysis. Londres: Routledge.

Hornberger, N. \& Chick, K. (2001). Co-constructing school safetime: Safetalk practices in peruvian and south african classrooms. En M. Heller \& M. Martin-Jones (Eds.), Voices of authority: Education and Linguistic Difference. Westport: Ablex Publishing.

Luyks, Aurolyn (1998). Discriminación sexual y estrategias verbales femeninas en contextos escolares bolivianos. Género en los Andes (pp. 33-69). Lima: PUCP.

Matos, J. (2004 [1984]). Desborde popular y crisis del Estado. Veinte años después. Lima: Fondo Editorial del Congreso del Perú. 
Ministerio de Educación del Perú (2004). Reglamento de Educación Básica Alternativa. Lima: Ministerio de Educación del Perú.

Montero, Carmen, Oliart, P., Ames, P., Cabrera, Z. \& Uccelli, F. (2001). La escuela rural: modalidades y prioridades de intervención. Lima: Ministerio de Educación.

Montoya, R. (1990). La escuela y el mito civilizatorio de Occidente. En R. Montoya (Ed.), Por una educación bilingüe en el Perú: reflexiones sobre cultura y socialismo. Lima: Mosca Azul.

Mujica, J. (2005). Las primeras batallas: poder y transgresión en la escuela primaria. Lima: Grade.

Oliart, P. (2011). Políticas educativas y la cultura del sistema escolar en el Perú. Lima: Instituto de Estudios Peruanos y Tarea.

Orlove, B. (1993). Putting race in its place: Order in colonial and postcolonial Peruvian geography. Social Research, 2(60), 301-336.

Street, J. \& Street, B. (2004). La escolarización de la literacidad. En V. Zavala, M. Niño-Murcia \& P. Ames. (Eds.) (2004). Escritura y sociedad. Nuevas perspectivas teóricas y etnográficas. Lima: Red para el desarrollo de las Ciencias Sociales en el Perú.

Trapnell, L. (2011). ¿Diversificar o interculturalizar el currículo? En S. Frisancho, M. Moreno, P. Ruiz Bravo \& V. Zavala (Eds.), Aprendizaje, cultura y desarrollo. Una aproximación interdisciplinaria. Lima: PUCP.

Torres, J. (1998). El curriculum oculto. Madrid: Ediciones Morata.

Tovar, T. (Ed.) (2002). Puertas Abiertas. Consulta Nacional de Educación (Tomos 1 y 2). Lima: Ministerio de Educación.

Vargas Ugalde, N. (2014). La noche de la literacidad escolar: prácticas letradas en una escuela pública del programa de Educación Básica Alternativa para estudiantes jóvenes y adultos en Lima (Tesis de licenciatura). Lima: PUCP.

Vásquez, A. \& Martínez, I. (1996). La socialización en la escuela: una perspectiva etnográfica. Barcelona: Paidós.

Willis, P. (1977). Learning to labour. How working class kids get working class jobs. New York: Columbia University Press.

Zavala, V. (2002). (Des)encuentros con la escritura. Lima: Red para el Desarrollo de las Ciencias Sociales en el Perú.

(2012). Dilemas ideológicos en torno a la educación intercultural bilingüe: El caso de la lectura en quechua. Revista Peruana de Investigación Educativa, 4, 77- 104.

Zavala, V. \& Córdova, G. (2010). Decir y callar. Lenguaje, equidad y poder en la universidad peruana. Lima: PUCP.

Zavala, V., Mujica, L., Córdova, G. \& Ardito, W. (Eds.) (2014).Quichwasimirayku. Batallas por el quechua. Lima: PUCP.

Zavala, V., Niño-Murcia, M. \& Ames, P. (Eds.) (2004). Escritura y sociedad. Nuevas perspectivas teóricas y etnográficas. Lima: Red para el Desarrollo de las Ciencias Sociales en el Perú. 